\title{
A Simulation Study on the Risk Assessment of the Modernization of Traditional Sports Culture Based on a Cellular Automaton Model
}

\author{
Minhang Liu (D), ${ }^{1}$ Gang Li, ${ }^{2}$ Qianhui Zhang, ${ }^{3}$ Xiaoyi Xu, ${ }^{4}$ and Ruijie Liu ${ }^{5}$ \\ ${ }^{1}$ School of Public Management, Shandong University of Finance and Economics, Jinan 250014, Shandong, China \\ ${ }^{2}$ School of PE, Shandong University of Finance and Economics, Jinan 250014, Shandong, China \\ ${ }^{3}$ Jinan Secondary Vocational School of Technology, Jinan 250000, Shandong, China \\ ${ }^{4}$ School of Architecture and Urban Planning, Shandong Jianzhu University, Jinan 250101, Shandong, China \\ ${ }^{5}$ School of Software, Qufu Normal University, Qufu 273165, Shandong, China \\ Correspondence should be addressed to Minhang Liu; sdufelmh@163.com
}

Received 16 August 2020; Revised 22 September 2020; Accepted 1 October 2020; Published 16 October 2020

Academic Editor: Xinguang Zhang

Copyright (C) 2020 Minhang Liu et al. This is an open access article distributed under the Creative Commons Attribution License, which permits unrestricted use, distribution, and reproduction in any medium, provided the original work is properly cited.

\begin{abstract}
Based on the cellular automaton model, the risk of modernization of traditional sports culture is evaluated and measured by using the methods of documentary, interview, and simulation experiments. The results of simulation experiment show that, in the complex system of the modernization of traditional sports culture, the mutation factors will lead to the sudden decline of the survival suitability of traditional sports culture and the risk of being unable to evolve; in the process of the modernization of traditional sports culture, there are two kinds of risks: self-extinction of dying out and self-extinction of homogenization; risk of dislocation failure lies in the government's support policies; risk of imbalance between fitness and scale allocation lies in modernity of derivative traditional sports culture. Countermeasures. The government should measure the environmental carrying capacity of the development of traditional sports culture in the region according to the regional resource situation; on this basis, heterogeneous sports activities are selectively introduced, the vitality of local traditional sports culture is enhanced, and the policy support and intervention time are reasonably controlled, to promote the balanced allocation of the suitability and scale of the derivative traditional sports culture.
\end{abstract}

\section{Introduction}

The traditional sports culture is the root and soul of sports development in China. But in the process of modernization, the soil on which the traditional sports culture depends has undergone great changes and is facing the risk that it may bring a survival crisis. The risk sources of traditional sports culture are diverse and complex, and different scholars also reveal and define the risk from different aspects. From the perspective of social transformation, national traditional sports culture faces the risk of lack of modern factors, elimination of living environment, and shrinking of target groups [1]; from the perspective of change, the four levels of material, institution, custom, and thought and value are inevitably facing crisis [2]; in the aspect of inheritance and development, the modernization of traditional sports culture faces many contradictions, such as nationalization and globalization, regionalization, and nationalization [3]. As far as current research is concerned, there is a lack of scientific measurement and intuitive quantitative research for the specific risk factor such as government policy. Moreover, the government policy has more countermeasures to deal with the risk, and there is less research on the risk brought by the policy itself, which provides the space for this research, that is, using cellular automata as the basis for constructing the risk assessment model, and based on explaining the composition and classification of cellular automata, the government policy is chosen as the key control parameter to carry out the simulation experiment of the risk of modernization of traditional sports culture and to measure and 
identify the risk categories to provide a reference for the formulation of accurate development policy.

\section{Simulation Experiment of Traditional Sports Culture Modernization Risk Based on Cellular Automata}

\subsection{Introduction of Cellular Automata Model}

2.1.1. Physical Definition of Cellular Automata. From a physical point of view, cellular automaton is a dynamic system that demonstrates a system that evolves in discrete time dimensions within a cellular space composed of cells with discrete, finite states, according to certain local rules [4].

In cellular automata, space is divided into several cells by a certain regular grid. Each cell in these regular grids is called a cell, and the value of each cell has a fixed limit; that is, it can only be taken in a set of finite discrete states. All cells must follow the same rules in their evolution. A large number of cells constantly update their self-state according to the rules of local evolution. Cellular automata can follow a series of evolutionary rules rather than strictly defined physical equations or functions. The model of cellular automata is similar to the "field" which can produce close action in traditional physics, which can be said to be a discrete model of "field" [5].

The basic idea of cellular automata is to use a large number of simple cells, thick through simple operation rules (that is, determined local evolution rules), in discrete time and space to continue to run, and then simulate the complex and rich various phenomena, providing effective model tools for the study of the overall behavior and complex phenomena of the system, and has been widely used in the research fields of economics, sociology, and other disciplines since its emergence [6].

2.1.2. Composition of Cellular Automata. The cellular automata model consists of four parts: cellular, cellular space, cellular neighbors, and cellular evolution rules. By mathematical definition, cellular automata can be understood as being composed of a cellular space and a transformation function scheduled for that space [7].

(1) Cellular. Cell, or unit, is the most basic unit of composition that can be calculated in cellular automata. Cells are all dispersed on lattice points in discrete 1,2, or multidimensional Euclidean spaces [8]. Strictly speaking, each cell must have its own state value at a fixed time, thus forming a state set, which can be a binary form of $\{0,1\}$ or a discrete set of $\{\mathrm{s} 0, \mathrm{~s} 0, \mathrm{~s} 1, \ldots, \mathrm{s} n\}$ integer form.

(2) Cellular Space. Cell space refers to a set of spatial lattice points distributed by cells. In theory, cellular space can be Euclidean space of arbitrary dimension, but the main research is 1-dimensional and 2-dimensional cellular automata (2-dimensional cellular automata model is used in this paper).

Geometric Division of Cellular Space. For 1-dimensional cellular automata, it is obvious that there is only one division of its cellular space, while in 2-dimensional cellular automata, its cellular spatial structure mainly involves three mesh arrangement modes: triangle, square, and regular hexagon. And this topic uses a square grid.

Boundary Conditions in Cellular Space. Even the most advanced computer simulation technology cannot complete the automata evolution experiment of an infinite grid. Data simulation must require the system to be finite and bounded. For the cell on the boundary, we can generally consider adding the boundary condition to the cell space. The general condition mainly has the fixed boundary, that is, adding the "virtual cell" of the fixed state outside the boundary of the cell space so that the boundary looks complete; the periodic boundary, in the 2D grid, mainly the left and right boundary and the upper and lower boundary, needs to be connected separately; there is also a kind of heat insulation boundary, adding "virtual cell" outside the boundary of the cell space, and the state determination of these "virtual cell" needs certain rules, which can give them the state of the boundary cell with which they are adjacent. This topic adopts fixed boundaries.

(3) Cellular Neighbors. The cellular and cellular spaces only define the static part of the cellular automata model. The cellular automata need to be equipped with corresponding evolution rules to carry out dynamic evolution. These rules are all defined in the local range, that is, the state of cellular at the next moment, depending on its state at the current moment, and the state of its neighbor cell. Therefore, the neighbors of the cell must be determined first before given the cell rules. In the $2 \mathrm{~d}$ cellular automata model, the commonly used neighbor structure is von Neumann and Moore type, and this subject is adopted by von Neumann.

The upper, lower, left, and right adjacent four cells of a cell are the neighbors of the cell. Mathematics is defined as

$$
N_{\text {Neumann }}=\left\{v_{i}=\left(v_{i x}, v_{i y}\right):\left|v_{i x}-v_{o x}\right|+\left|v_{i y}-v_{o y}\right| \leq 1, \quad\left(v_{i x}, v_{i y}\right) \in \mathrm{Z}^{2}\right\},
$$

where $v_{i}=\left(v_{i x}, v_{i y}\right)$ denotes the neighbor cell and its coordinates and $\left(v_{o x}, v_{o y}\right)$ is the coordinates of the central cell.

(4) Rules of Cellular Evolution. According to the cellular state at present and its neighbor state, the dynamic function of determining the state of the cell at the next time is generally called the state transfer function [9]. For the given cell $i$, all of its neighbors $N_{i}$, the state of cellular $i$ recorded at $t$ time is $S_{i}^{t}$ so the state of the entire neighborhood can be recorded as $S_{N_{i}}^{t}$. The state transfer function can be recorded as $F$. As a 
result, every cell $i$ has $S_{i}^{t+1}=F\left(S_{i}^{t}, S_{N_{i}}^{t}\right)$. It can be said that the cellular evolution rule is the key to cellular automata and the dominant factor. The reasonable selection of the evolution rule is related to the success of a cellular automata model.

\subsection{Construction of Risk Simulation Model of Traditional} Sports Culture Modernization Based on Cellular Automata. Taking the key control parameters of traditional sports culture development, government policy as the research object, the following CA models are established:

$$
S^{t}=f\left(\Omega, S^{t-1}, \phi(N), A, R^{t}\right) .
$$

The meaning of the elements in formula (2) is as follows:

(1) $\Omega$ mark cell space, $\Omega=\{(i, j), \quad i, j=1,2, \ldots, N\}$.

(2) $S^{t}(i, j)$ and $S^{t-1}(i, j)$ are the strategies for labeling cells at $x$ and $y$ times, which is defined as follows:

$$
S^{t}(i, j)=\left\{\begin{array}{l}
0 \\
1 \\
2
\end{array}\right\},
$$

where 0 represents no policy involvement in project development, 1 represents the macropolicy guidance of the sports culture represented by a project, and 2 represents the sports culture of a project.

(3) $\phi(N)$ labels the neighborhood of intercellular interaction, and this paper uses the von Neumann model.

(4) $R^{t}(i, j)$ represents the winning rate of $(i, j)$ cell at $t$ time, also known as the fitness of the cell.

During the development of traditional sports culture, when the government policy begins to intervene in the development of culture, the behavior derived from traditional sports culture is the game behavior of policy and cultural development system; that is, the derivative state of traditional sports culture will have a certain influence after receiving the influence of different policies. In $\Omega$, the formula for calculating the suitability of internal cells $R$ is

$$
R^{t}(i, j)=\left[1-\left(\sum \frac{S^{t-1}(i, j)}{N}\right)\right]\left(1-L^{t-1} \sigma\right)\left(1+E^{t-1}\right) .
$$

For the convenience of simulation, this study stipulates that, from the beginning of the reform and opening up in 1989, the influence of the policy on the development of traditional sports culture is set a fixed constant:

$$
R^{t}(i, j)=0.1\left(1-L^{t-1} \sigma\right)\left(1+E^{t-1}\right) .
$$

The relationship between the elements in formulae (4) and (5) is explained as follows: $\sum S^{t-1}(i, j)$ is expressed as the number of ecological traditional sports cultural items under the influence of policy at $t-1$ time, that is, the number of relevant policies selected as 1 or 2 in the cellular space, and this study will be one of the indicators to measure the risk of sports culture modernization.

$\mathrm{N}$ indicates the number of all traditional sports cultural items (including original and new ecology) under the influence of policy.

$1-\left(\sum\left(S^{t-1}(i, j)\right) / N\right)$ is expressed in a certain time and space, the survival of ecological traditional sports, and cultural projects due to the dependence on policy caused by their own growth block.

$1-L^{t-1} \sigma$ reflects the game competition influence of all kinds of traditional sports culture items under the influence of policy.

$L^{t-1}$ denotes the number of cells in the neighborhood space belonging to the evolution state of national traditional sports culture at $t-1$ time.

$\sigma$ represents the effect of competition between cells on the cell in the neighborhood space.

$E^{t-1}$ is a remarkable feature and one of the important parameters in the modernization of traditional sports culture.

Generally, we regard the scale change in derivative traditional sports culture is affected by policies such as urban and rural S-shaped curve change; that is, at the beginning, the response to the policy is slow, and then, it enters the rapid development period. With the enhancement of adaptability, the policy stimulus tends to decline. Therefore, the influence of policy parameters conforms to the typical diffusion curve, and the calculation formula used is as follows:

$$
E^{t-1}=\frac{1}{\left(1+a \exp \left(-b \sum E^{t}(i, j)\right)\right)}
$$

In (6), at time $t$, the diffusion rate affected by policy is the parameter of the above model. Parameter $a$ determines the position of the curve, and parameter $b$ determines the shape of the curve. The larger the value of $b$, the steeper the curve is, which means that the response speed of traditional sports cultural projects to policies is very fast, and the derivative scale is larger. Therefore, this study is mainly through the study of parameter $b$ to explore the influence of policy parameters on the development scale of traditional sports culture.

(5) $f$ is the state transition rule function.

Each traditional sports culture item may have a random variation on the derivative route. Because of the policy influence, different projects have a certain 
contraction strategy, that is, either new or choose to die out.

\subsection{Simulation Steps}

Step 1: $t=0$ assigns an initial value to the threshold for the number of experiments $T$, maximum policy tolerance $N$, threshold $r, a, b$, and $\sigma$ threshold value. At the moment of $t=1$, at the center of the cell space $(20 * 20$ matrix), there exists a $2 * 2$ matrix; that is, the derivative formed under the influence of the policy of the beginning of the traditional sports culture project.

Step 2: end if $t>T$;

Step 3: $E^{t-1}$ from formula (6);

Step 4: find $R^{t-1}$ and $R^{t}$ from formulae (4) and (5);

Step 5: calculation $S^{t}(i, j), \bar{R}^{t}(i, j), \sum s^{t}(i, j)$, using rules $f$;

Step 6: calculation $S^{t+1}(i, j)$, using rules $f$;

Step 7: $t=t+1$, step 2 .

All the above steps can be programmed by MATLAB to complete the simulation calculation.

2.4. Simulation Diagram. In the simulation process of this study, the cell network of $20 * 20$ is mainly adopted, the number of simulations is specified as $T=70, N=(1 / 2)(20 * 20)$, and $r=0.3$, and then different initial values are given to $b$ and $\sigma$; the following series of simulation diagrams are obtained, as shown in Figures 1-8.

(1) $a=60, \sigma=(1 / 8), b=0.2$, and the simulation results are shown in Figures 1 and 2.

(2) $a=60, \sigma=(1 / 8), b=0.8$, and the simulation results are shown in Figures 3 and 4 .

(3) $a=60, \sigma=(1 / 2), b=0.2$, and the simulation results are shown in Figures 5 and 6 .

(4) $a=60, \sigma=(1 / 2), b=0.8$, and the simulation results are shown in Figures 7 and 8.

\section{Interpretation of Result}

From the results of the above eight diagrams, we can analyze and obtain four significant risks of the cultural development of the derivative traditional sports culture project.

3.1. Risk 1: Extinction Self-Extinguishing. When $a=60, \sigma=$ $(1 / 8)$ (see Figures 1 and 2); when the policy parameter $b=0.2$, that is, the impact of policy is not strong. At the same time, the average suitability of the policy to the traditional sports culture has changed from the initial intense response to zero. This phenomenon is called extinction self-extinction. The most likely reason for this is that the policy is not in line with the market demand, which leads to the traditional sports culture.

The project's derivative ecology appeared "survival suffocation" and then "revived from the dead" with the strong support of the government. There is a growing gap in development. At this time, the policy measures are not conducive to the health of traditional sports culture development.

As the policy parameters $b=0.8$, that is, the policy changes, the average, fitness, and development scale of the traditional sports culture evolution ecology gradually appear as local peak, but after the local peak appears, there is no big change, which indicates that the support strategy can only support it to the current high level, but the support fatigue phenomenon. The possible reasons are as follows: first, the traditional sports culture in the evolution of the "ride" behavior, the project because of regional rules, and no one to support innovation, and this situation will lead to the entire project's evolutionary die out. Secondly, maintaining the leading edge of technology, leading innovation projects in traditional sports culture will be taken strict knowledge protection measures by the higher authorities to prevent the spread of innovation technology; the signing of confidentiality agreements for the descendants and the registration of trademarks and patent rights in the form of expression are all-powerful measures to protect the development of local projects, but these measures can often also become resistant to their development.

3.2. Risk 2: Homogenization Self-Extinguishing. As the traditional sports culture system has not died out, when the policy parameters change from 0.2 to 0.8 , the scale of development of the traditional sports culture is shrinking at $x=(1 / 2)$ (Figures 5-8), which shows that when the government's support policy is strengthened, the external economic diffusion effect of traditional sports culture is obvious, and the traditional sports culture system has already manifested competitive advantages such as the scale of participating artists, the rapid spread of influence, and the strong regional brands. Of course, this cannot say which policy is the most reasonable, and only after the market test can we determine the good policy, but this time, often many homogeneous sports in the system have died out or are in a state of extinction, and the emergence of homogenization is self-extinguishing.

3.3. Risk 3: Risk of Dislocation Failure in Government Support Policies. The simulation results show that when the market competition is in a smooth state, the policy support of the government or the competent department does not promote the evolution of the traditional sports culture modernization but only affects the upper limit of the system scale of the traditional sports culture. Suppose the government support policy is the main control parameter. Figures 1-4 show that when the policy is not strong, $b=2$; the average fitness $\bar{R}^{t}(i, j)$ is $0.3-0.7$ when the evolutionary risk effect of all sports culture modernization in the region spreads slowly, while the traditional sports culture is affected by the policy, the development scale $\sum S^{t}(i, j)$ fluctuation range is $60-120$; when the policy is strong, that is, $b=0.8$, the fluctuation range of the average fitness $\bar{R}^{t}(i, j)$ is $0.3-0.9$, while the development scale $\sum S^{t}(i, j)$ fluctuation range of traditional sports culture is $20-120$. It can be seen that when the social 

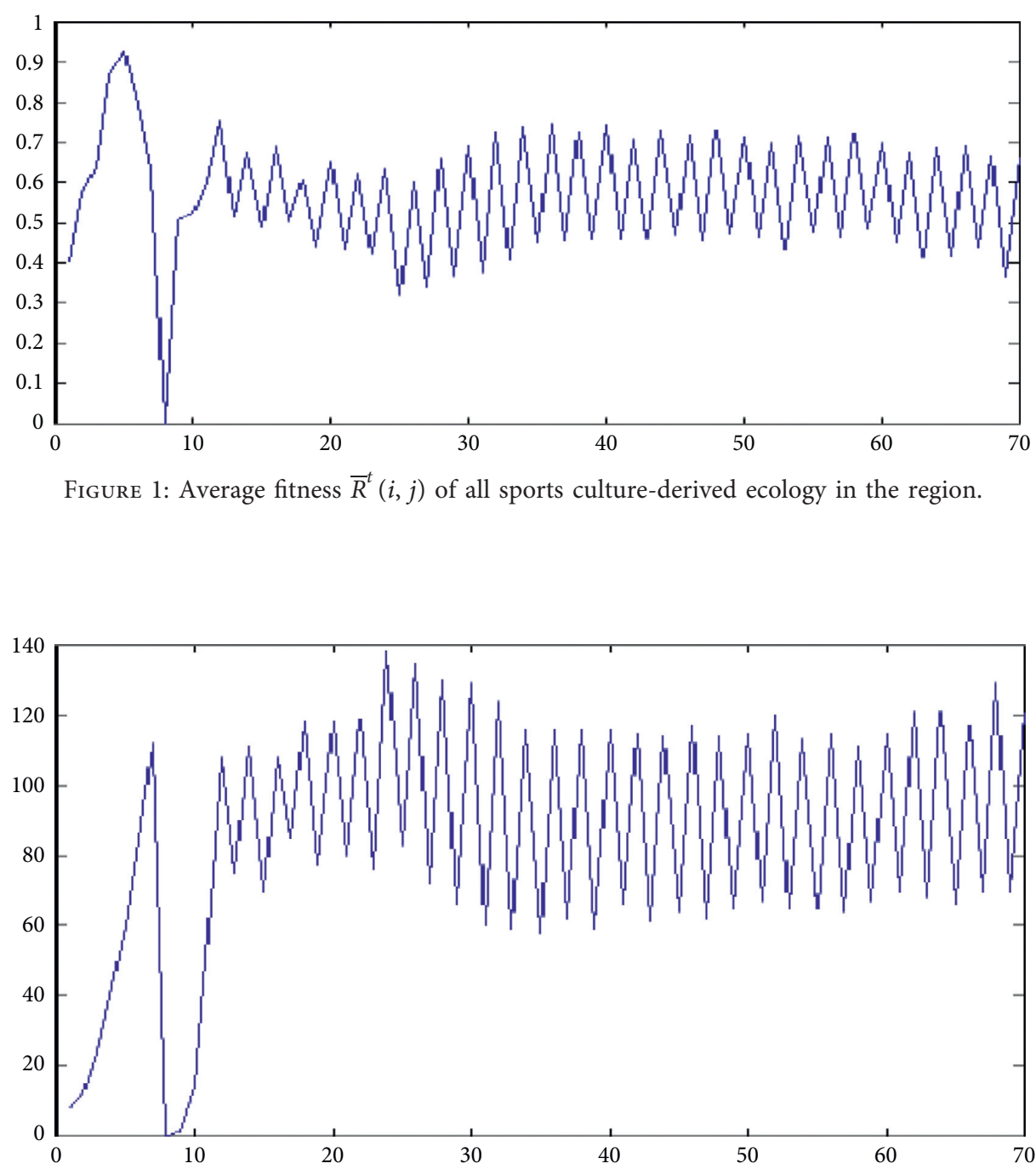

FIGURE 2: $\sum S^{t}(i, j)$ of development scale of derivative ecology after being affected by policy.

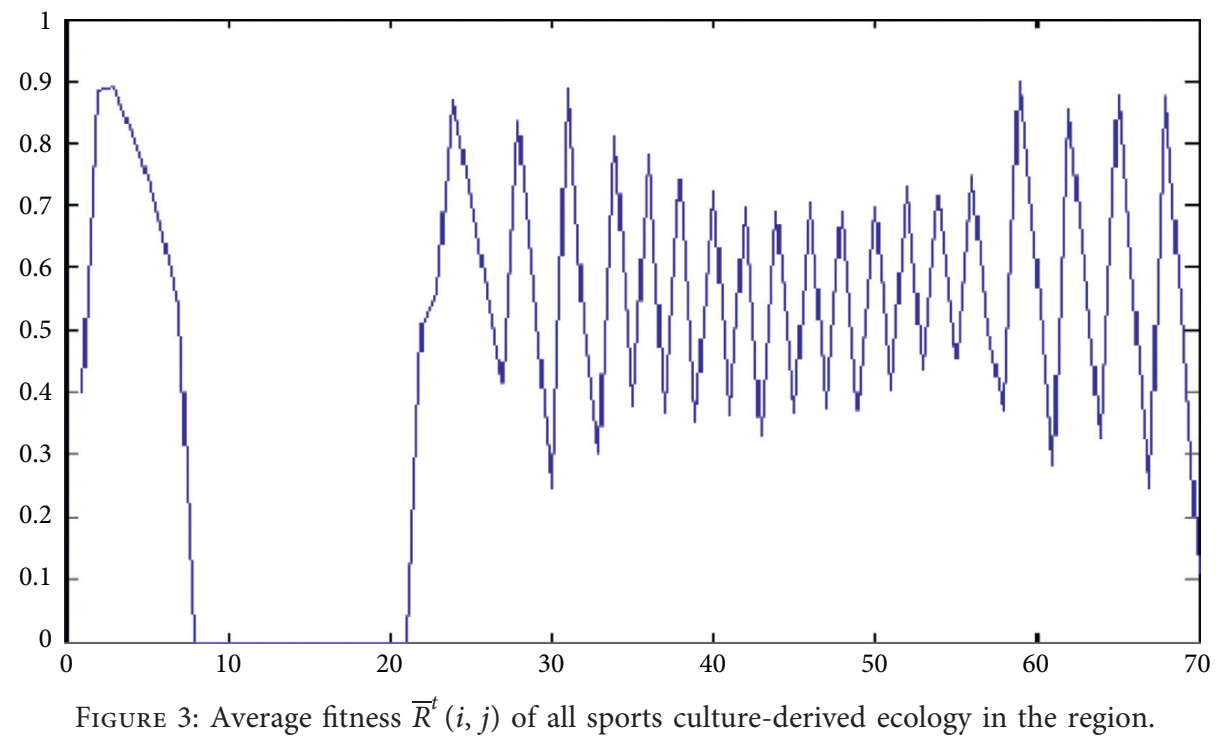




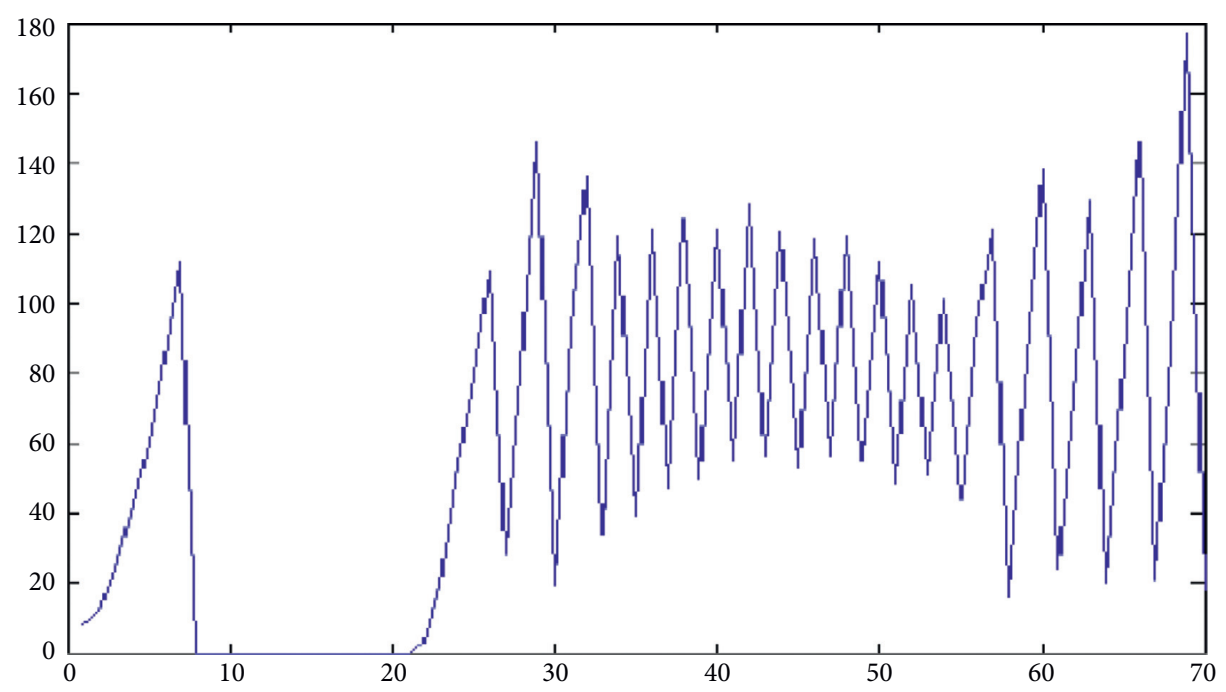

FIGURE 4: $\sum S^{t}(i, j)$ of development scale of the derivative ecology after being affected by policy.

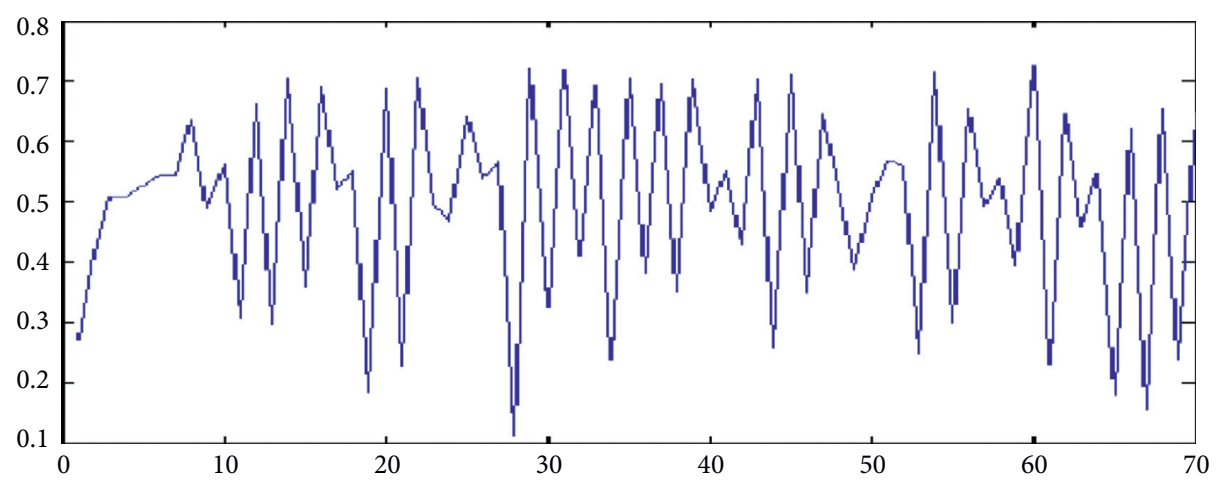

Figure 5: Average fitness $\bar{R}^{t}(i, j)$ for all sports culture-derived ecology in the region.

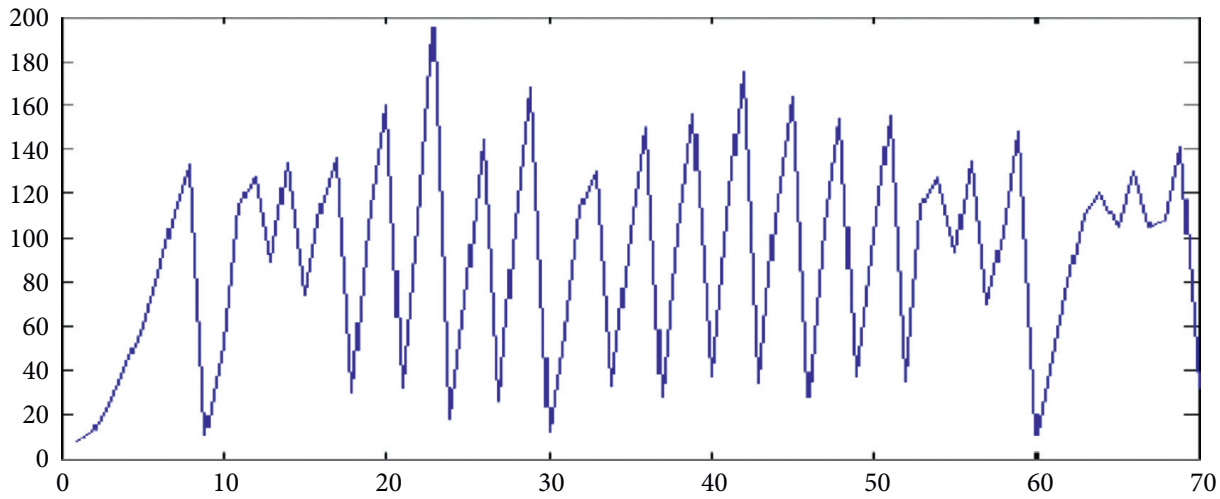

FIGURE 6: $\sum S^{t}(i, j)$ of development scale of the derivative ecology after being affected by policy.

environment changes, mainly in the environment of fierce market competition, the support of the government has little influence on the development scale derived from the traditional sports culture, which shows that blindly introducing policies to support will make the policy miss the best time to intervene, which will not only reduce the efficiency of the policy but also affect the self-adjustment of the traditional sports culture.
3.4. Risk 4: Unbalance of Fitness and Scale Allocation of Traditional Sports Culture. When $a=60$ and $\sigma=(1 / 2)$, we can see that when the government policy is not strong, $b=0.2$, the average fitness of the items in the traditional sports culture system is $0.1-0.7$, and the scale of the traditional sports culture is $20-160$; if the government policy is strong, $b=0.8$, the average fitness of the items in the traditional sports culture system is $0.2-0.8$, and the scale of the 


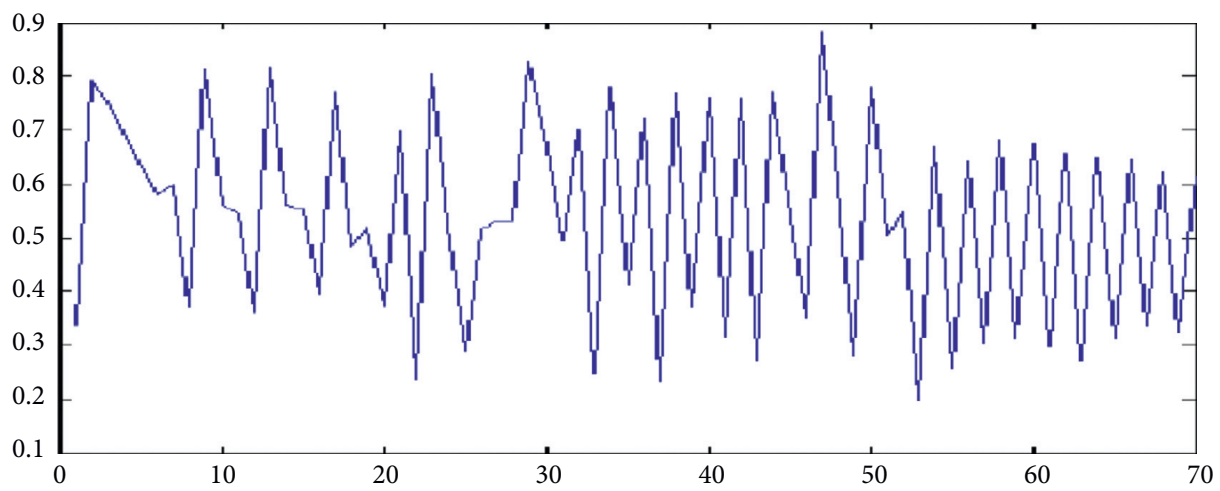

Figure 7: Average fitness $\bar{R}^{t}(i, j)$ for all sports culture-derived ecology in the region.

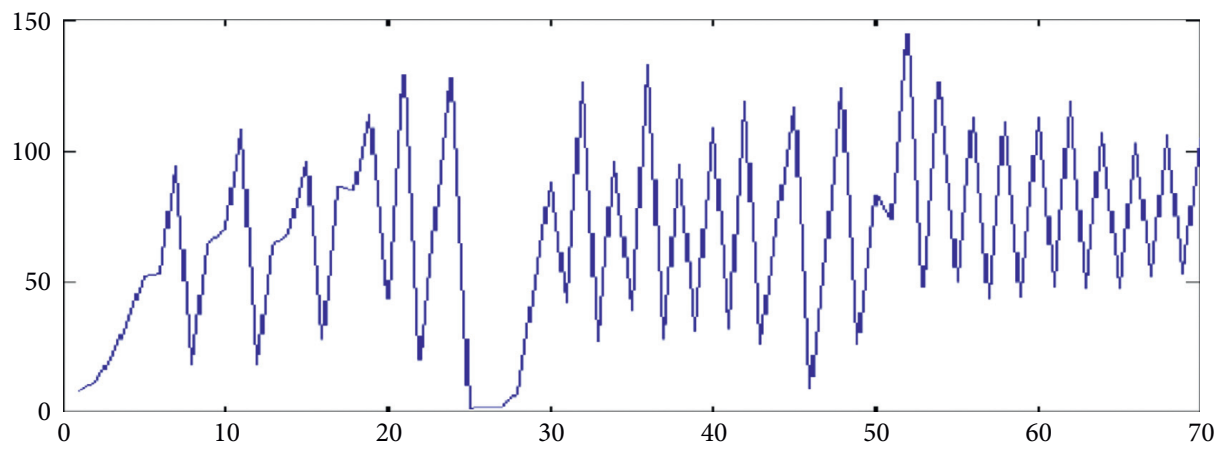

Figure 8: $\sum S^{t}(i, j)$ of development scale of the derivative ecology after being affected by policy.

traditional sports culture is $0-150$. This shows that the policy will enhance the suitability of the components in the traditional sports culture project system, but it will inhibit the development of the traditional sports culture scale. Therefore, whether to improve the conformity of the traditional sports culture or to enlarge the scale of its modern development tests the management wisdom of relevant departments. Partially emphasizing conformity or development scale may bring great challenge to traditional sports culture.

\section{Risk-Avoiding Strategy for Modernization of Traditional Sports Culture}

4.1. Actively Introducing Heterogeneous Sports. To avoid the phenomenon of self-extinguishing, the government should actively introduce as many heterogeneous sports as possible to stimulate the innovation and development of local traditional sports. Of course, in the process of development, the government cannot forcibly change its development structure, but through the external environment to change its internal factors, thus causing its evolution. To complete the modernization and evolution of local traditional sports, the competent authorities should take the search for sports with heterogeneity and stimulation as one of the important tasks.
4.2. Measuring the Environmental Carrying Capacity of the Development of Traditional Sports Culture in the Region. It is suggested to do a good job of statistics and track the scale of sports culture development after the policy release and to predict the environmental carrying capacity of traditional sports development in the region before the new policy release. Evaluate the suitability of the policy, use the CA model, measure the development scale of traditional sports culture, and prevent the risk of self-extinguishing, unbalance, and even failure. Therefore, in the development of the traditional sports culture system, the measurement of environmental carrying capacity is necessary and crucial.

4.3. Policy Support and Timing of Intervention. According to the current situation of inheritance and protection of traditional sports culture in China, government guidance and support are still the main forces to promote its modernization [10]. But the simulation experiment shows that the government policy support strength and the intervention time, should according to the external environment situation, realize the precision support, thus enhancing the policy utility. So when does government support work on traditional sports culture? When the strength of policy support changes from 0.2 to 0.8 , the average fitness of sports culture development ecology has been improved to some extent, but 
the threshold of development scale fluctuation is different. Therefore, when the government formulates the support policy to the traditional sports culture, it must first measure key control parameters (economic and geographical conditions) in its evolution process, so it is possible to determine the timing of policy intervention and better control the influence [11].

\subsection{To Promote the Balanced Allocation of Fitness and Scale of} Traditional Sports Culture in Derivative State. Because fitness and scale are the important factors that affect the development of ecological traditional sports culture, it should be determined according to the external environment and development stage of traditional sports culture [12]. It can be divided into the following situations: first, when the internal and external environmental resources of a certain region are limited and the competition between projects is fierce, the government should minimize the policy interference and let the different traditional sports in a free competition state; when the competition reaches a certain stage, the government chooses the opportunity to enter, support some projects, and improve the suitability of the project system; second, when the internal and external resources available in a certain region are abundant and the competition between projects is small, the government should strengthen the policy, on the one hand, improve the suitability of traditional sports culture, on the other hand, make full use of external resources to promote its scale development, and achieve the double promotion of suitability and scale; third, when the regional internal and external environmental resources are abundant and the project competition is more intense, the government should make full use of the resources to promote the development of the project scale at this time [13].

\section{Conclusion}

According to the research needs, this paper selects the government policy as the key control parameter to simulate the four risks in the process of modernization of traditional sports culture, which is of great significance for the protection and inheritance of national culture. In fact, in the era of big data, the "cloud computing" of the modernization development of traditional sports culture in the region is not difficult, and it is expected that soon, the modernization development of traditional sports culture projects can achieve the precise control of various influencing factors and develop towards the direction of human needs and hopes.

\section{Data Availability}

Data sharing is not applicable as no datasets were generated or analyzed during the current study.

\section{Conflicts of Interest}

The authors declare that there are no conflicts of interest regarding the publication of this paper.

\section{Authors' Contributions}

The study was carried out in collaboration among all authors. All authors read and approved the final manuscript.

\section{Acknowledgments}

The first author was supported by the Project of National Social Science Fund of China (NSSF) (no. 18BTY015). The second author was supported by Outstanding Youth Innovation Team of Institutions of Higher Learning in Shandong Province (no. 2019RWF014).

\section{References}

[1] J. Yin and N. Li, "Dilemma and enlightenment of modernization transformation of Chinese national traditional sports culture," Journal of Xi'an Institute of Physical Education, vol. 33, no. 1, pp. 88-93, 2016.

[2] X. Xu, Z. Lin, and M. Liu, "A study on the risk category of modernization of traditional sports culture," Shandong Sports Science and Technology, vol. 41, no. 1, pp. 7-10, 2019.

[3] Y. Bai and G. Wang, "Contradictions in the development of national traditional sports modernization," Sports Journal, vol. 1, pp. 62-64, 2005.

[4] M. Zeng, C. Wang, P. Liu, and L. Liang, "Path planning of mobile robot based on cellular automata," Journal of Nanchang University (Engineering Edition), vol. 34, no. 3, pp. 287-290, 2012.

[5] Ze Sun, B. Jia, and X. Li, "A study on interference mechanism of pedestrian and motor vehicles based on cellular automata," Journal of Physics, vol. 61, no. 10, pp. 76-83, 2012.

[6] G. Chen, B. Cai, and M. Li, "Cellular automata: an effective tool to explore the complexity of management systems," China Engineering Science, vol. 39, no. 1, pp. 28-32, 2007.

[7] X. Duan, C. Wang, X. Tian, and Q. Zhang, "A one-dimensional binary cellular automata behavior measure based on evolution entropy," Complex Systems and Complexity Science, vol. 9, no. 2, pp. 7-12, 2012.

[8] C. Li and L. Zhong, "A study on finite element method based on cellular automata," China Water Transport (Second Half Month), vol. 16, no. 12, pp. 95-98, 2016.

[9] F. Chen, Z. Cui, and D. Dong, "Research progress in cellular automaton simulation of microstructure evolution," Journal of Mechanical Engineering, vol. 51, no. 4, pp. 30-39, 2015.

[10] C. Huang and J. Li, "Social roots and solutions of cultural inheritance of village folk sports," Journal of Beijing University of Physical Education, vol. 41, no. 12, pp. 123-129, 2018.

[11] Z. Wang, N. Wang, F. Fei, and W. Tian, "Evolutionary behavior of cellular automata," Computer Applied Research, vol. 8, pp. 38-41, 2007.

[12] $\mathrm{Zi} \mathrm{Ming,} \mathrm{"Research} \mathrm{on} \mathrm{the} \mathrm{transmission} \mathrm{mode} \mathrm{of} \mathrm{national}$ traditional sports culture from the perspective of intangible cultural heritage protection," International Core Journal of Engineering, vol. 6, no. 5, p. 14, 2020.

[13] X. Xu, Z. Yang, L. Jin, and L. Hao, "Research on risk assessment of port project investment and construction," Journal of Coastal Research, vol. 9, no. 11, pp. 187-189, 2020. 